\title{
La(s) pobreza(s) desde el género. Una aproximación a la realidad chilena actual ${ }^{1}$
}

\author{
Poverty(ies) from a gender's perspective. An approximation to \\ the current Chilean reality
}

Andrea Baeza², Pamela Baeza ${ }^{3}$ V Victoria lbaceta ${ }^{4}$

\begin{abstract}
Resumen
El presente escrito analiza la pobreza en Chile. Las acepciones más tradicionales de corte económico, e incluso otras sociales surgidas posteriormente, presentan deficiencias a la hora de aprehender la complejidad de este fenómeno. Limitar la pobreza a la familia, desconociendo las asimetrías de género y de poder presentes al interior de ella y en la sociedad nos brinda una visión parcial de este problema social. Hay que mirar dentro de la "armonía del hogar"y reconocer, además de la carencia material e inmaterial, que existe una carencia temporal que afecta principalmente a las mujeres.
\end{abstract}

Palabras claves: pobreza - género - mujeres - desigualdad de tiempo - Chile.

\begin{abstract}
This paper analyzes poverty in Chile. The more traditional meanings are economic and even other social meanings subsequently emerged have deficiencies to grasp the complexity of this phenomenon. Limiting poverty to family, ignoring gender asymmetries and power relations within it and in society, it gives us a partial view of this social problem. We have to look into the "harmony of the home" and besides of to recognize the material and immaterial lack, that there is a temporary shortage affecting mainly to women.
\end{abstract}

Key words: poverty - gender - women - time inequality - Chile.

Fecha de recepción: 24 de abril de 2012

Fecha de aprobación: 1 de septiembre de 2014

PUNTO GÉNERO / 83 


\section{INTRODUCCIÓN}

El panorama de la pobreza e indigencia, tanto en Latinoamérica como en nuestro país ha sido alentador en los últimos años. En la última década del siglo pasado, casi la mitad de la población de la región se encontraba en situación de pobreza o pobreza extrema, mientras que en 2013 se ha podido reducir esta cuantía en un 20,5\% y 11,1\% respectivamente. Así, si en 1990 existían 204 millones de personas afectadas con esta condición, hace un año, las estimaciones dejaban fuera a 40 millones de personas a considerar bajo estos fenómenos durante dicho periodo. Todo esto, pese al fuerte impacto de la reciente crisis financiera internacional, donde la región mostraba signos de continuidad en La tendencia a la reducción de la pobreza que despegó en 2003 (CEPAL, 2010, CEPAL, 2013) (ver gráfico 1).

\section{Gráfico 1 AMÉRICA LATINA: EVOLUCIÓN (\%) DE LA POBREZA E INDIGENCIA. 1980-2013}

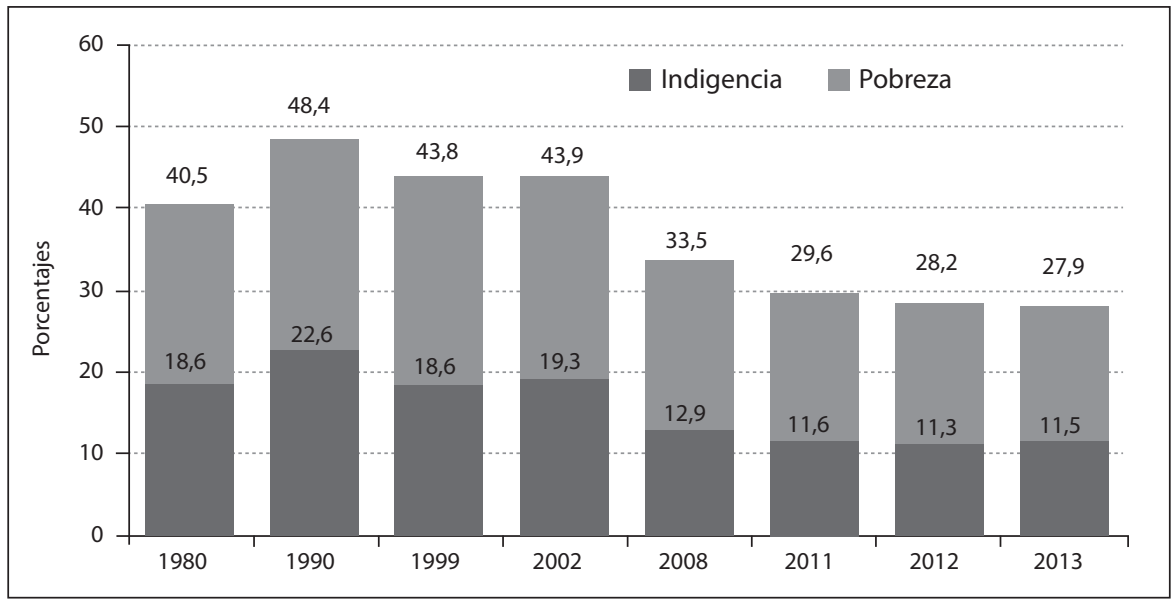

Fuente: CEPAL, 2013: 18.

La evolución de la pobreza desde los 90 en Chile ha sido similar, mostrando reducción en un 25\% hasta 2011. A partir de 1996, la disminución de estas cifras entre un 4\% y 5\% empieza a adquirir una variación menor, por debajo del 2\%. Recién en 2003 es posible retomar el descenso en más de 5 puntos porcentuales para 2006, que luego de un leve aumento de las cifras de pobreza a nivel nación, continúa la tendencia a la baja (ver Gráfico 2).

En dicho escenario, no obstante, es necesario ir más allá de los números y preguntarse ¿qué es lo que se entiende por pobreza?, ¿a qué y a quiénes afecta?

La importancia de clarificar cómo es definida la pobreza radica en el hecho de que diferentes acepciones de este concepto implican el uso de diversos indicadores para su medición, lo que puede conducir a la identificación de diferentes sujetos como 


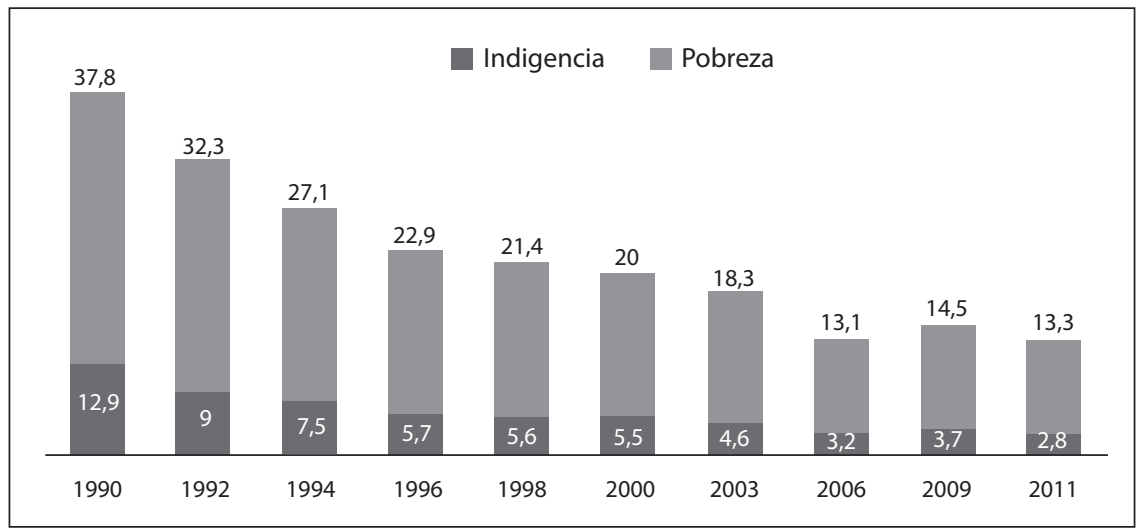

Fuente: Elaboración propia a partir de los datos del Ministerio de Desarrollo Social (2012b).

pobres, y de la mano, distintas soluciones políticas para la reducción de la pobreza (Ruggeri, Saith y Stewart, 2003: 1).

Existen distintos enfoques para comprender el fenómeno de la pobreza, que van desde las perspectivas más economicistas hasta las de carácter asistencial. Así, el enfoque monetarista -el más comúnmente utilizado- define la pobreza como "un descenso en el consumo o ingreso, en función de carencias o necesidades básicas insatisfechas, mediante indicadores como la ingesta de alimentos, el nivel de ingresos, el acceso a la salud, educación y vivienda" (CEPAL, 2004: 10). Sin embargo, y pese a la amplia difusión de este enfoque, quedan fuera una serie de elementos relativos a la pobreza y que son fundamentales para entender este fenómeno, como componentes psicosociales y culturales, y las dimensiones normativas, institucionales y cognitivas de los afectados (Arriagada, 2006: 3). De este modo, el monetarismo es insuficiente para comprender la complejidad de la pobreza, ya que solo se centra en el ámbito económico, desconociendo las múltiples dimensiones que presenta este problema, y porque examina hogares, excluyendo el hecho de que en algunos de estos haya miembros que sí puedan ser considerados como tales, ya que la distribución de los recursos en su interior no es igualitaria.

Aunque se han diversificado las perspectivas para abordar la pobreza, aún son precarias en cuanto no tematizan que las relaciones sociales no son neutrales, y los recursos mucho menos homogéneos para los distintos miembros del hogar. Por ello, se hace necesario analizar desde una perspectiva de género la pobreza. Esta no afectaría de igual modo a hombres y mujeres, tanto en el mismo hogar como fuera de él. En el primer caso, la pobreza se expresa diferenciadamente, según sea la conformación familiar (jefe o jefa de hogar), el acceso a recursos económicos, sociales y simbólicos de ellos y ellas y la distribución del tiempo. En el segundo, cabe señalar el ingreso y permanencia en el mercado laboral, la ocupación y niveles de empleo y las 
remuneraciones también son diferenciadas. El trasfondo en ambas situaciones, vendrían a ser las visiones esencialistas en torno a las mujeres, que las reducen a madres y esposas, por su rol reproductivo. De este modo, es menester "abrir la caja negra del hogar", que a simple vista parece una "unidad de intereses compartidos" (Hartmann, 2000: 19), y que encubre muchas veces situaciones de discriminación que perjudican a las mujeres, que constituyen obstáculos a la hora de superar la condición de pobreza.

Por consiguiente, se sostiene la necesidad de contemplar la pobreza y vulnerabilidad no solo en su dimensión colectiva en términos de hogares, sino en su dimensión individual, que sin pretensiones de sustituir a la primera, permita dar cuenta de la multicausalidad de este fenómeno social, así como también de la heterogeneidad de sus efectos en los sujetos y sujetas.

\section{POBREZA HOY... ¿Y MAÑANA? HACIA UN MARCO REFERENCIAL DEL CONCEPTO}

Al hablar de pobreza, necesariamente hay que hablar de vulnerabilidad, ya que son conceptos estrechamente vinculados. Mientras la pobreza refiere al estado de situación actual, la vulnerabilidad es el riesgo de padecerla o agravarla en el futuro.

Según Moser, "vulnerabilidad corresponde a la inseguridad del bienestar de los individuos, los hogares o las comunidades ante un ambiente que cambia" (1996: 2), ya sea en términos económicos, sociales, políticos o ecológico. Significa indefensión, inseguridad, exposición a riesgos y estrés inducidos por acontecimientos socioeconómicos extremos (Baranda y Egenau, 2004), cuyo impacto de trauma afecta a individuos y sus familias, debilitando sus propios recursos y estrategias para enfrentar tales sucesos. Se trata, entonces, de un estado donde las personas pierden bienestar, en parte, por la variabilidad del ingreso económico

Pobreza, en cambio, ha sido más difícil de aprehender por la multiplicidad de visiones que la delimitan y definen lo que constituye una "buena vida". Más allá del desafío conceptual que ello suscita, diferentes enfoques se traducen en diferentes estrategias para medir la pobreza, lo que tiene efectos políticos significativos. En la siguiente sección se presentan y discuten algunos de estos enfoques.

\section{a) Enfoque monetarista}

Es el mayormente difundido, a la hora de entender este fenómeno. Dentro de este, pobreza sería el "descenso en el consumo o ingreso, y que toma como base una línea de pobreza" (CEPAL, 2004: 10) donde el supuesto clave, según Ruggeri, Saith y Stewart (2003), es que con las herramientas adecuadamente diseñadas, indicadores monetarios uniformes pueden tomar en cuenta toda la heterogeneidad relevante entre los individuos y sus situaciones ${ }^{5}$.

Traducción propia 
La fuerza de este enfoque, desarrollado desde el siglo XIX con los estudios de Booth y Rowntree ${ }^{6}$, reside en que lo que se desprende del indicador monetario construido: (1) la pobreza es una condición objetiva (por lo que podía ser medida), (2) su apreciación es externa, y (3) debe ser definida respecto de circunstancias individuales y conductas y no como un fenómeno social.

Los indicadores monetarios se han considerado un método conveniente basado en datos que son ampliamente disponibles para identificar a quienes son pobres en diferentes dimensiones fundamentales, no solo relacionados con la falta de recursos monetarios (medición indirecta), sino también a la nutrición, salud, entre otros (medición directa).

La medición indirecta de la pobreza implica el cálculo de los ingresos per cápita, cuando estos no alcanzan la adquisición de lo que se conoce como la canasta básica de alimentos ${ }^{7}$ (Ministerio de Desarrollo Social, 2013), la que equivale a un "contenido calórico y proteico [que] permite satisfacer un nivel mínimo de requerimientos nutricionales por persona al mes" (ibíd., 2014).

La medición directa sugiere el método de necesidades básicas insatisfechas (NBI) en donde "se elige una serie de indicadores censales que permiten constatar si los hogares satisfacen o no algunas de sus necesidades principales" (Feres y Mancebo, 2001: 8). Es aquí donde se construyen mapas de pobreza ${ }^{8}$, a partir de carencias según las mediciones de las"características de la vivienda", como por ejemplo, el tipo de materiales, acceso a agua potable, a sistema de eliminación de excretas o número de cuartos; y a "ciertos rasgos demográficos del hogar" (ibíd.), como el número de miembros, la asistencia escolar de los menores, la edad, el nivel educativo y la condición de ocupación principal del sostenedor del hogar.

Así, a partir de ambas se han establecido las línea de pobreza e indigencia, cuyos criterios de demarcación entre quiénes son pobres o extremadamente pobres"se definen en relación a mínimos de satisfacción de necesidades básicas, en el caso de la pobreza, o alimentarias, en el caso de la indigencia" (Ministerio de Desarrollo Social, 2013: 30).

Hay que señalar que ha habido ajustes metodológicos en la estandarización de la medición de pobreza, como ha sido el empleo de los datos de consumo en vez de

$6 \quad$ Booth realizó su investigación en el este de Londres en 1887 no consultando directamente a los pobres, sino utilizando informantes. Con esta información categorizó a la población en ocho clases sociales donde cuatro representaban diferentes grados de pobreza. Aunque para Ruggeri, Saith y Stewart (2003: 8) su clasificación fue más allá de una mera identificación monetaria de los pobres, abarcando cuestiones más sociológicas como las condiciones del hogar y del trabajo. En esa línea, Rowntree definió una línea de pobreza estimando los requerimientos para una dieta nutricionalmente adecuada junto con una estimación de las necesidades de ropa y renta.

7 Su valor económico es determinado por el Instituto Nacional de Estadísticas (INE) en función del Índice de Precios al Consumidor (IPC). Actualmente, equivale a \$40.611, y ha ascendido \$6.658 desde julio del año 2011 (Ministerio de Desarrollo Social, 2014)

8 Instrumento que responde a la necesidad de localizar los lugares más pobres del país 
los de ingresos, ya que evita fluctuaciones de corto plazo en la renta y en el acceso a ciertos recursos y porque el ingreso es una variable difícil de registrar, que "adolece de subregistros sistemáticos y presenta proporciones significativas de no respuesta" (Arriagada, 2006: 3). Sin embargo, los datos de ingreso son los más frecuentemente usados para delimitar la línea de pobreza.

Un problema, es que no considera las transferencias indirectas y subsidios del Estado. Además, la valoración de los distintos componentes de ingreso o consumo se realizan considerando los precios del mercado por lo que se requiere la identificación de cuáles son los productos necesarios para vivir y cuantificar los productos que no son valorados por el mercado. Otra dificultad es que las líneas de pobreza con frecuencia cortan intervalos modales de ingreso, y no ciertamente donde estarían los verdaderos pobres.

En ese sentido, para Ruggeri, Saith y Stewart (2003) este enfoque está fundamentalmente dirigido a los logros individuales, las interacciones e interdependencias sociales son consideradas solo desde el punto de vista mecánico de la escala adecuada de los recursos del hogar para tener en cuenta las diferentes estructuras familiares ${ }^{9}$. Esta estandarización olvida otras dimensiones sobre las que se extiende este fenómeno y homogeniza las características particulares en la forma de experimentar la pobreza por parte de los individuos. Por último, el monetarismo desconsidera que las mediciones tienden a ser muy sensibles a las situaciones de coyuntura, como la inflación, el desempleo y la ideología política de gobierno.

\section{b) Enfoque de las capacidades}

Retomando el "protagonismo de los pobres" y la libertad desde una perspectiva positiva, Amartya Sen en los noventa atiende el empoderamiento de los más desfavorecidos para despertar sus potencialidades, capacidades y productividades.

Esta nueva teorización de la pobreza apunta a la puesta en práctica de estas aptitudes fundamentales, lo que el autor denomina "funcionamiento", entendiendo por ello la realización concreta susceptible de ser llevada a cabo por cada persona para "permanecer vivo y gozar de una vida larga y saludable, reproducirse y transmitir su cultura a las generaciones futuras". Los "funcionamientos", entonces, están vinculados a la "ampliación de la gama de opciones de una población" (ibíd.), en la medida en que todos deben tener oportunidades de llevar una vida satisfactoria. Existirían funcionamientos que son elementales, como "la adecuada alimentación, tener vivienda o gozar de buena salud" (Parker, 2002: 9), y otros más complejos, como estar integrado y ser respetado, ante los cuales se hace necesario reconceptualizar el ingreso como un medio y las necesidades, como las que posibilitan el desarrollo de aquellas capacidades.

No obstante, los reparos a este enfoque son que la manera en que define la pobreza sigue siendo estrictamente individual, y parte del supuesto que la pobreza es

$9 \quad$ Traducción propia.

88 / PUNTO GÉNERO 
una "limitación en las capacidades" que habría que entrar a desarrollar. Por el contrario, no es que los pobres no tengan capacidades, "sino que están dotados de una cantidad limitada de capacidades potenciales", las que están condicionadas, precisamente, "por la estructura de oportunidad que ofrece la sociedad" (ibíd.).

\section{c) Enfoque participativo}

Este enfoque incluye a las personas afectadas por la pobreza en su evaluación a partir del informe del Banco Mundial (2000), "Las voces de los pobres. ¿Alguien puede oírnos? Además de la definición más clásica en cuanto a la privación de activos para adquirir alimentos, vivienda y gozar de buena salud, y rescatando la visión de hombres y mujeres en 20 países en vías de desarrollo, se resaltan aspectos psicológicos como la percepción de falta de poder en la toma de decisiones, de autonomía y sobre todo, la dificultad de reproducir culturalmente ciertas prácticas de sus comunidades y la estigmatización de quienes son pobres, como son la humillación e inferiorización para defenderse de abusos y explotaciones.

Será, entonces, con colaboración activa de los pobres en la medida que sean ellos mismos, a partir de su propia realidad social, los que definan su pobreza, y puedan abrir caminos para superarla a partir del desarrollo de capital humano. Ello dependerá de las condiciones particulares de cada persona, como así también del contexto histórico, político y cultural de cada país (UNFPA, 2005).

Sin embargo, esta perspectiva de la pobreza puede caer en hiperresponsabilización de los sujetos de pobreza acerca de su condición. Sin querer negar el carácter subjetivo de esta, es preciso señalar que no basta exclusivamente con el ímpetu de los individuos para contrarrestar la pobreza, sino que con "los mecanismos que operan al interior de los hogares para mantener o aumentar su bienestar o para no caer en mayores niveles de pobreza" (Arteaga, 2007: 14) dentro de la estructura de oportunidades que los permiten.

\section{d) Enfoque Activos, Vulnerabilidad y Estructura de Oportunidades (AVEO)}

Dentro de la heterogeneidad de las situaciones de pobreza existen "grados variables" de propiedad, control e influencia sobre los "activos, capitales o recursos" 10 y estrategias para movilizarlos (Filgueira, 1999 citado en Arteaga, 2007: 155). Serán sobre aquellos, la probabilidad de seguir o caer en la pobreza. Aquí cobra relevancia el "aprovechamiento de las oportunidades que ofrece el medio a través del mercado, el Estado o la sociedad" (Katzman y Filgueria, 1999: 8), los que pueden brindar el acceso a bienes y servicios incidentes en movilizar "trabajo, capital humano, capital social, además de los recursos materiales" (Arteaga, 2007: 158). Todo ello con el propósito de permitir o facilitar el uso de recursos propios o generar nuevos a los miembros de los hogares.

10 A saber, está la mano de obra, la vivienda, las relaciones familiares, el mismo capital social o la infraestructura económica. 
La fuerza del AVEO reside en que permite vincular el análisis micro de los hogares con aspectos macro de la institucionalidad en lo que respecta al estudio de la pobreza y observar, no desde una perspectiva estática, la capacidad de adaptación a los diversos cambios del entorno en relación con las oportunidades y recursos tanto materiales como inmateriales (activos) que pueden aprovechar y movilizar los afectados.

Por otro lado, y teniendo en cuenta que las oportunidades brindadas no son constantes y varían en unidades territoriales ${ }^{11}$ y momento histórico de las naciones, una salvedad es el rol preponderante del mercado sobre el Estado y la sociedad en la actual sociedad chilena. Precisamente, la reducción de las atribuciones del Estado "por medio de la transferencia al mercado y a la sociedad civil de gran parte de las funciones de integración, protección y cobertura de la seguridad social" (Katzman y Filgueria, 1999: 14) impactan en la producción, distribución y uso de los activos para la movilidad e integración social.

\section{e) Enfoque de exclusión}

El concepto exclusión se acuñó en los países industrializados para describir la marginalización y privación producidos incluso en aquellas naciones con servicios de bienestar integral, y hace referencia a un proceso mediante el cual los individuos o grupos son total o parcialmente excluidos de una participación plena en la sociedad en que viven (Fundación Europea, 1995 citado en Ruggeri, Saith y Stewart, 2003: 20).

En estricto rigor, la exclusión es el proceso gradual de quebrantamiento de vínculos sociales y simbólicos, "cuando individuos o comunidades sufren una combinación de diversos problemas vinculados", a saber, "la exclusión laboral; exclusión económica; exclusión institucional; aislamiento social; exclusión cultural y territorial" (Baranda y Egenau, 2004: 22), la que puede presentarse en reiteradas formas y momentos.

La conveniencia de este enfoque, es que permite centrarse en elementos como la edad, discapacidad o categorías raciales y étnicas; y con ello, poder incorporar una agenda política diferente a las de los enfoques individualistas. Considera también la multidimensionalidad y amplitud de la pobreza, donde los mecanismos de exclusión son de distinta índole y las personas o grupos pueden verse privados en más de una dimensión ${ }^{12}$.

11 Sobre la centralidad del contexto espacial en los que residen los individuos que se encuentran en situación de pobreza, el territorio es una construcción social donde se concentran y se puede acceder a distintos activos. Este es un condicionador y al mismo tiempo está condicionado tanto por el "conjunto organizado (formal o informal) y complejo de potencialidades naturales, humanas e institucionales y de sistemas de asentamiento poblacional" (MIMDES, 2005: 8), como por los intereses, identidades y la cultura que atraviesan a las particularidades de las comunidades. De esta concepción se desprende, por lo tanto, que no es igual la pobreza rural a la urbana, ni en la capital respecto de las regiones.

12 Por ejemplo, en los mercados de trabajo, bienes y servicios, en la participación y la representación; en la identidad, conocimiento valores y/o ubicación geográfica. 
Desde esta perspectiva, la centralidad será entonces atender las dinámicas excluyentes de las oportunidades de participación social y de los recursos, en los diversos individuos y/o determinados grupos sociales en términos de familia, comunidad y Estado. Dichas oportunidades están sujetas en gran parte a la distribución del ingreso en la sociedad, y que en Chile implica una distribución desigual pese al crecimiento económico de los últimos años, lo que se ha traducido en "inequidad de los beneficios [... y y las oportunidades" (Bengoa, 2001: 52). Con ello se tiene un sector que concentra la propiedad de activos y otro que no goza de protección social (Molina, 2005). En otras palabras, un grupo que participa ampliamente en sociedad, y otro más amplio y que con mayor o menor grado, está excluido.

\section{f) Enfoque del capital social}

Esta óptica más reciente, también apunta a visibilizar aspectos más intangibles de la pobreza. El capital social consiste en el "conjunto de recursos a disposición de los integrantes de una red durable de relaciones" (Bourdieu, 1985 en Arriagada, 2006), o como diría Kliksberg (2000), el manejo del código simbólico-social a disposición de los individuos, grupos y comunidades, que les permitirían aumentar el bienestar material, facilitar el ejercicio de la ciudadanía y a la vez, constituirse en un mecanismo de control social, en la medida que sus bases son la reciprocidad, confianza y la cooperación social.

El capital social es el recurso impalpable primordial desde donde se tejen las relaciones de apoyo y se construye el escenario protector para las personas en situación de pobreza y vulnerabilidad social. En efecto, si se aspira a una intervención integral sobre estas complejidades, desarrollar el capital pasa por considerar elementos que no se desarrollan aisladamente, como son la memoria social, identidad, vecindad, estima, amistad, prestigio, entre otros asociados al establecimiento y fortalecimientos de redes.

Si bien este enfoque resalta las relaciones sociales como posibilidad de obtención de beneficios orientados a la superación de la pobreza, a veces omite las relaciones de poder entre y al interior de dichos grupos (entre ellas, las que se establecen entre hombres y mujeres), así como también las relaciones clientelares que existen entre las instituciones y las organizaciones sociales de base.

\section{MIRAR LA POBREZA DESDE EL GÉNERO}

Retomando los elementos principales de los enfoques mencionados, la Comisión Económica para América Latina y el Caribe (CEPAL) define pobreza como:

"un sindrome situacional, asociado al infraconsumo, la desnutrición, las precarias condiciones de vivienda, bajos niveles educacionales, malas condiciones sanitarias, inserción inestable en el aparato productivo, actitudes de desaliento y anomia, poca participación en los mecanismos de integración social y quizá la adscripción a una escala particular de valores diferenciada en alguna medida del resto de la sociedad" (2004: 12). 
A pesar de la evidente extensión del término "pobreza"con la incorporación de otras dimensiones más allá del ingreso para comprenderla, se mantiene intacto un supuesto susceptible a poner en tela de juicio: la igualdad de pobreza para hombres y mujeres. Aunque la anterior definición entregada por esta institución es más amplia, aun así es insuficiente al invisibilizar la desigual distribución de recursos y necesidades entre los individuos de distinto género y edad, y la expresión de relaciones de poder que posicionan distintamente a sus miembros. Por ello, es menester redefinir la pobreza desde la perspectiva de género.

\section{a) Desigualdad en los quehaceres}

Son, precisamente, algunas condicionantes de la vulnerabilidad como el acceso al trabajo formal y remunerado, el nivel de ingreso, las decisiones sobre este, el nivel educativo, la jefatura de hogar y los usos de tiempo, que están "vinculados a cuestiones de género" principalmente por "expectativas sociales" las que "limitan o posibilitan de forma diferente a mujeres y hombres" (García, 2010: 10) para superar la pobreza.

Partimos del hecho que la pobreza es heterogénea, puesto que es vivida de forma distinta en hombres, mujeres y niñas y niños. Asimismo, las estrategias para contrarrestar y superar las circunstancias inherentes y externas de la pobreza presente o en el futuro, no impactan de igual manera entre sus miembros, puesto que imponen una carga desigual entre quienes conforman un hogar, siendo las mujeres particularmente afectadas por las atribuciones sociales dentro de este último.

Desde un enfoque de género, es posible desplazar la usual categorización social de pobreza desde los hogares hacia los individuos, al mostrar el carácter subordinado de la participación de las mujeres en la sociedad, por ejemplo, "en las posibilidades de acceso a la propiedad y al control de los recursos económicos, sociales y políticos". También reconoce la peculiar relación entre el trabajo remunerado y no remunerado, donde "la creciente incorporación de las mujeres al mercado del trabajo no ha significado una incorporación paralela de los hombres a las actividades domésticas y de cuidado de los hijos, ancianos, enfermos u otros familiares". En efecto, "las mujeres siguen asumiendo el trabajo doméstico y el cuidado de los hijos de manera casi exclusiva" (Arriagada, 2010: 3). Más específicamente, "Ios esposos de mujeres que trabajan fuera de casa, no pasan más tiempo en quehaceres domésticos que los esposos de aquellas que no lo hacen" (Hartmann, 2000: 36). Según la última encuesta de uso de tiempo (EUT) realizada en el Gran Santiago, las mujeres que realizan las labores del hogar prácticamente duplican en proporción a los hombres y en cuanto a las labores de cuidado de otros en este escenario, los triplican (ver Gráfico 3).

En esta asimétrica distribución de quehaceres, entonces, es posible distinguir la persistencia de una mentalidad tradicional de larga duración, que a las mujeres "coloca en una situación de inferioridad ante los hombres, ligando su potencial reproductivo con la atribución de tareas reproductivas" (Arriagada, 2010: 5). El perjuicio reside en que como las mujeres terminan adquiriendo mayores cargas de trabajo al interior del hogar, se 


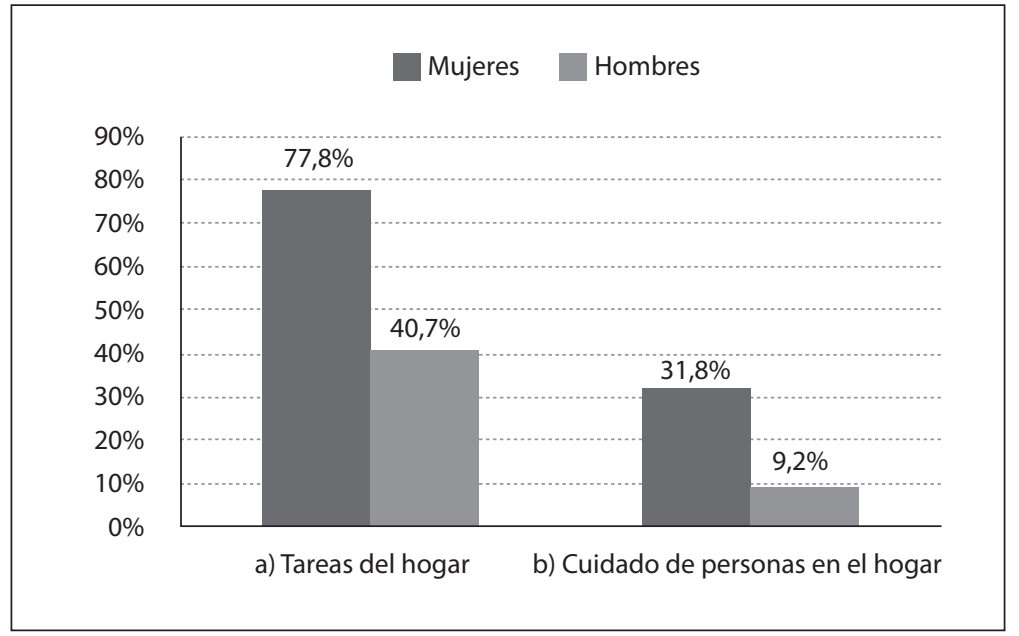

Fuente: Elaboración propia a partir de los datos del INE (2009).

ven limitadas sus capacidades para "aprovechar oportunidades de desarrollo y coloca a sus hogares en condiciones de mayor vulnerabilidad" (Moser, 1996 citado en García, 2010: 21).Como lo indica la autora, "Ia familia es todavía el terreno principal en el que los hombres ejercen su poder" (Hartmann, 2000: 33), "está configurada por el patriarcado y por el capitalismo, por lo que la estructura social descansa en la división desigual del trabajo" (ibíd.: 20).

\section{b) Otra pobreza eminentemente femenina}

A partir de lo anterior, podemos afirmar que existe otra pobreza que atañe casi exclusivamente a las mujeres: la "pobreza de tiempo".

Como lo indica Hartmann, "la producción del hogar [... ] es claramente mayor a un trabajo de jornada completa" En prácticamente todos los países de occidente ocurre que las esposas que trabajan fuera de la casa, lo hacen bastante más horas todos los días que los esposos o las amas de casa a tiempo completo, sobre todo si cuidan a sus familiares (Hartmann, 2000: 36). Esto remite a la doble o tercera jornada que deben enfrentar las mujeres que reciben ingresos, a saber: el trabajo asalariado, el trabajo doméstico y el cuidado de otros. En breve, la participación laboral remunerada de las mujeres no trastoca los roles asignados históricamente entre los géneros.

Lo anterior supone familias biparentales. Pero cuando hay solo uno de estos, también existen diferencias en cuanto a las jefaturas de aquellas y más aún en lo que refiere a hogares pobres. Si analizamos quienes son los principales sostenedores de hogar hasta el 2011, se observa que las familias a cargo de un solo progenitor 
en nuestro país, también presentan una fuerte discrepancia si es que se examinan según género.

De acuerdo con las cifras recientes del Ministerio de Desarrollo Social (2013), en el quintil más bajo, un 32,4\% corresponde a familias compuestas por solo un progenitor y casi un 10\%, a aquellas con ambos progenitores. En términos totales, empero, resulta que es eminentemente predominante la jefatura femenina en aquellos de tipo monoparental y la masculina, en los biparental. Como es posible apreciar a continuación, hace más de una década, un 16\% de las jefas de hogar sacaba adelante a sus familias sin compañía de sus parejas. Hace dos años, esta cifra aumentó un 7\%, siguiendo la tendencia al alza desde 2000. Para el caso de los hombres, el fenómeno es inverso durante este mismo periodo. Ellos figuraban inicialmente en un 70\% dentro de hogares biparentales, el que si bien ha descendido un 16\% hasta 2011, continúa siendo muy superior respecto de los ínfimos porcentajes de hogares monoparentales en que están insertos (ver Gráficos 4 y 5).

\section{Gráfico 4}

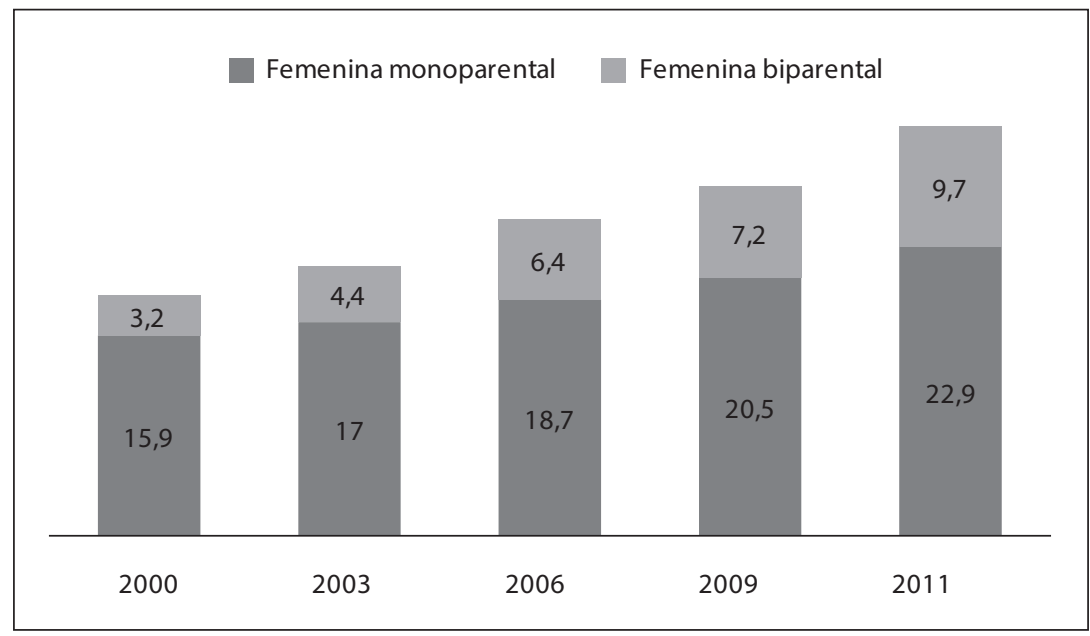

Fuente: Elaboración propia a partir de los datos del Ministerio de Desarrollo Social (2013).

La última encuesta CASEN (2011) revela que los pobres no indigentes e indigentes se redujeron en un 5\% y 24\% en relación con el año 2009 respectivamente. No obstante, este fenómeno mantiene rostro de mujer, donde más de la mitad de los hogares con jefatura femenina se encuentran en situación de pobreza (51\%) y pobreza extrema (55\%). Esto es lo que se conoce como la feminización de la pobreza.

Lo anterior, y más allá de los números de familias que son lideradas o no por mujeres, lo podemos vincular a una diferencia aún más profunda: la desigual distribución de las tareas domésticas al interior del hogar, y con ello, los usos diferenciados del tiempo para mujeres y hombres. En efecto, hay una serie de aspectos circundantes al trabajo doméstico. Por un lado, en los hogares con jefatura masculina, estas labores suelen 


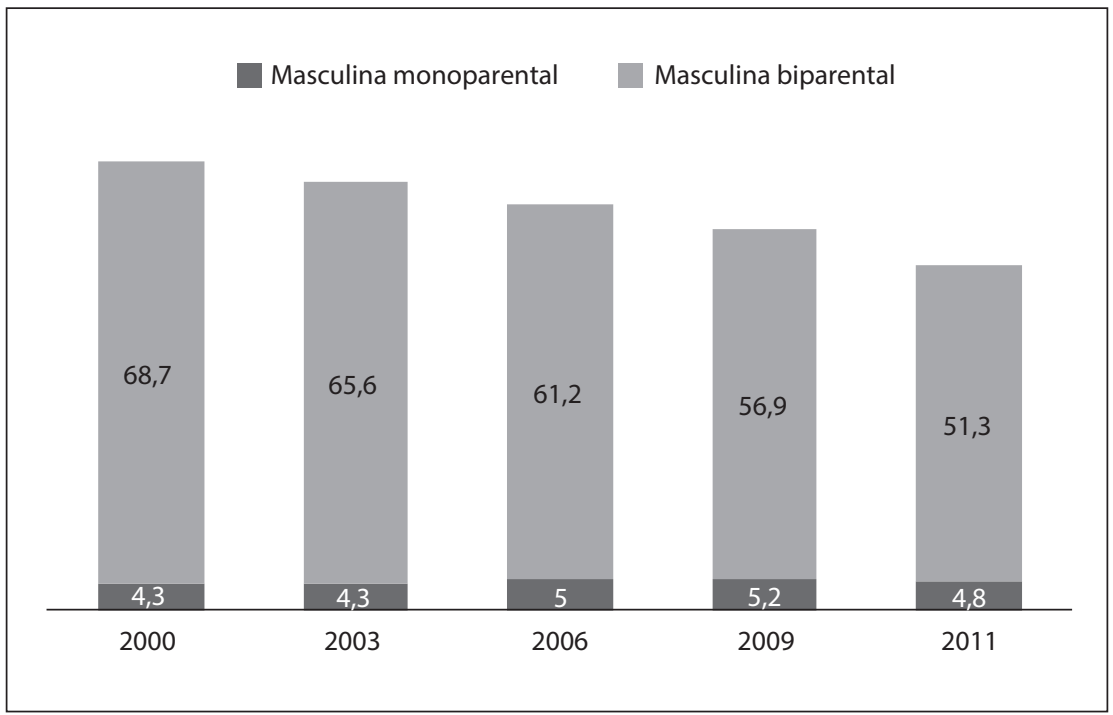

Fuente: Elaboración propia a partir de los datos del Ministerio de Desarrollo Social (2013).

estar exentas de gastos monetarios (de pagarle a alguien externo, ya que lo realiza principalmente la esposa o conviviente) y temporales (si fuese él quien lo realizase). De este modo, los hombres están más libres para realizar otras actividades referentes al ocio, entretención o similares. En los hogares de jefaturas femeninas, por otro, "es más probable que la mujer destine [sus] ingresos a estos servicios [si le paga a un externo] o que aumente su jornada de trabajo total" si los asume (CEPAL, 2004: 25).

Según es posible de apreciar en la "cultura de las tareas domésticas", es la mujer la protagonista de estos quehaceres, ya sea de manera única o con apoyo, pero quien destina más del 70\% de su tiempo a esta jornada ahoraria (ver Gráfico 6).

Esto va de la mano con la denominada "economía del cuidado", vale decir, con el hecho que en ellas recaiga la responsabilidad y responsabilización de la continuación, mantención y reparación de otros cuerpos como vienen a ser el de sus hijos o sus padres, en tanto adultos mayores. Ambas forman parte de la reproducción social que, desarrollada en el ámbito privado y exiliada de los cálculos economicistas, permite dotar al Estado de funcionarios, soldados y ciudadanos, y por tanto, es indispensable a la hora de hacer funcionar la economía de un país. Este subsidio gratuito a la nación que realizan las mujeres desde "la cuna hasta la tumba", en la medida que "la gran mayoría de las mujeres casadas [y las también solteras] se ocupan de un trabajo que resulta vital aunque no se pague, sin el cual sus maridos [u otros familiares] no podrían realizar trabajo remunerado y sin el cual la nación no podría continuar" (Aguirre y Scuro Somma, 2010: 12, citado en CEPAL, 2012a: 23). Esto se correlaciona con la histórica división sexual del trabajo, en donde a la mujer se le remite de manera exclusiva al hogar, es decir, al 


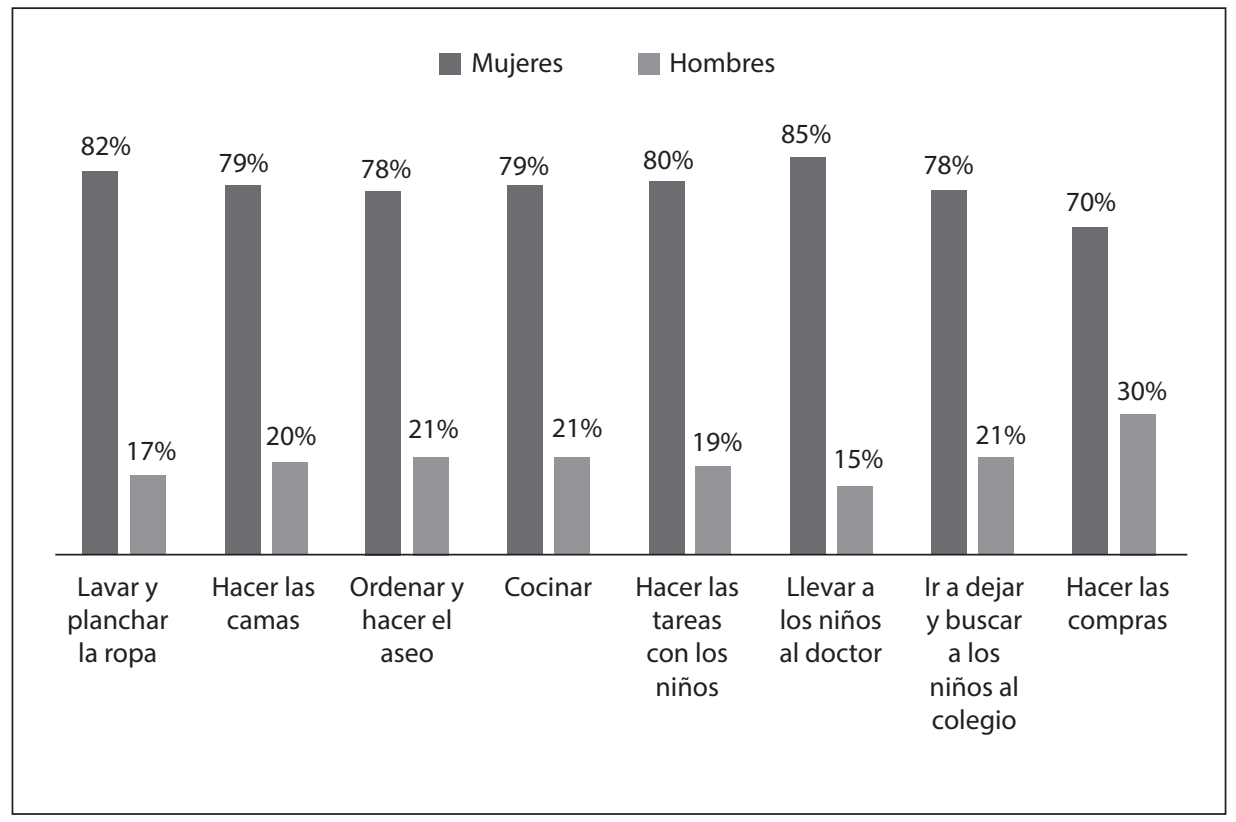

Fuente: PNUD, 2010: 73.

mundo privado, pero cuando logran desempeñarse en lo público, no implica que se desliguen de su rol tradicional doméstico y de cuidado.

Según el estudio de "Valorización del Trabajo no Remunerado" encargado por el Servicio Nacional de la Mujer (SERNAM, 2009), en promedio, las personas destinan un $42,78 \%$ del tiempo diario a necesidades biológicas y cuidados personales como dormir, comer, asearse y otros; un 22,01\% a actividades remuneradas o de capacitación; un 16,53\% corresponde a tiempo comprometido, vale decir, dedicado a actividades domésticas no remuneradas y voluntariados; y el 18,68\% restante viene a ser el tiempo libre. Sin embargo, si comparamos entre los géneros continúan las asimetrías. Precisamente en estas últimas dos categorías, las mujeres dedicarían 224,73 minutos o lo que equivale a de 3,7 horas más que los hombres a los quehaceres del hogar, y tienen 34,36 minutos menos de tiempo libre respecto de ellos (ver Gráficos 7 y 8).

Menor tiempo libre se traduce en su sociabilidad, en tanto la "desigualdad de género tiene una de sus fuentes en la reclusión de las mujeres en el ámbito del hogar". De igual modo, cabe destacar la diferencia en cuanto al sentido de dicho tiempo libre para ellos y para ellas. Mientras los hombres lo significan como un tiempo autónomo del de la jornada remunerada, las mujeres "lo experimentan de manera simultánea a otros tiempos" (PNUD, 2010: 78), por ejemplo, ver televisión mientras se cocina, o mientras se plancha. 


\section{Gráfico 7 TIEMPO COMPROMETIDO SEGÚN GÉNERO (PORCENTAJE)}

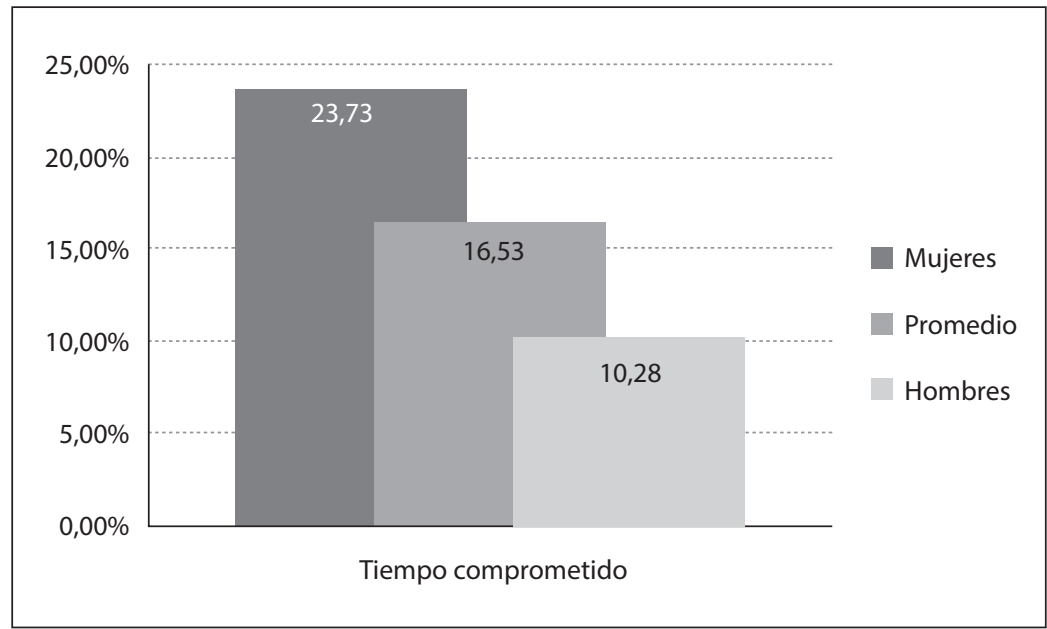

Fuente: Elaboración propia a partir de datos del SERNAM (2009).

\section{Gráfico 8 TIEMPO LIBRE (\%) SEGÚN GÉNERO}

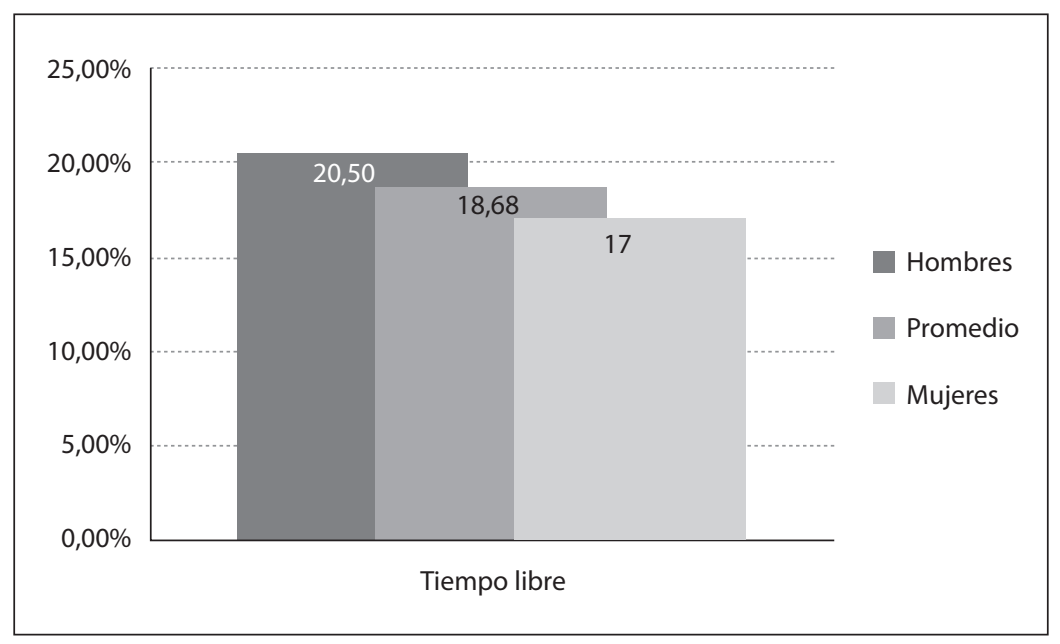

Fuente: Elaboración propia a partir de datos del SERNAM (2009). 
En ese sentido, se tiene que factores de género efectivamente se traducen en mayor vulnerabilidad para las mujeres, cuando arrastran "elevados requerimientos de trabajo doméstico y cuidado de menores [... . enfermos, discapacitados y adultos mayores" (Damián, 2013: 145). En términos prácticos, les significa menos tiempo libre ${ }^{13}$ y mayor tiempo comprometido, lo que no solo se traduce en una precarización del tiempo destinado a sí mismas, sino que además un deterioro físico (¿y por qué no? mental14) que les afecta especialmente en la constitución de sujetos autónomos económicamente.

Cabe añadir a lo anterior, el obstáculo para salir de la pobreza que significa la dificultad de acceder a mejores empleos. En nuestro país, dentro del primer quintil, para 2011, solo el 39,9\% de las mujeres entre 25 y 59 años participaba del mercado laboral, en contraste, con el 78,6\% de los hombres de entre 29 y 64 años (Ministerio de Desarrollo Social, 2013). Esto se correlaciona con que la educación castiga más fuertemente a las mujeres que a los hombres en la región latinoamericana, donde ellos obtienen mayor empleabilidad en todos los niveles, siendo solo a los 13 o más años de instrucción que la brecha se reduce, pero no desaparece (ver Gráfico 9).
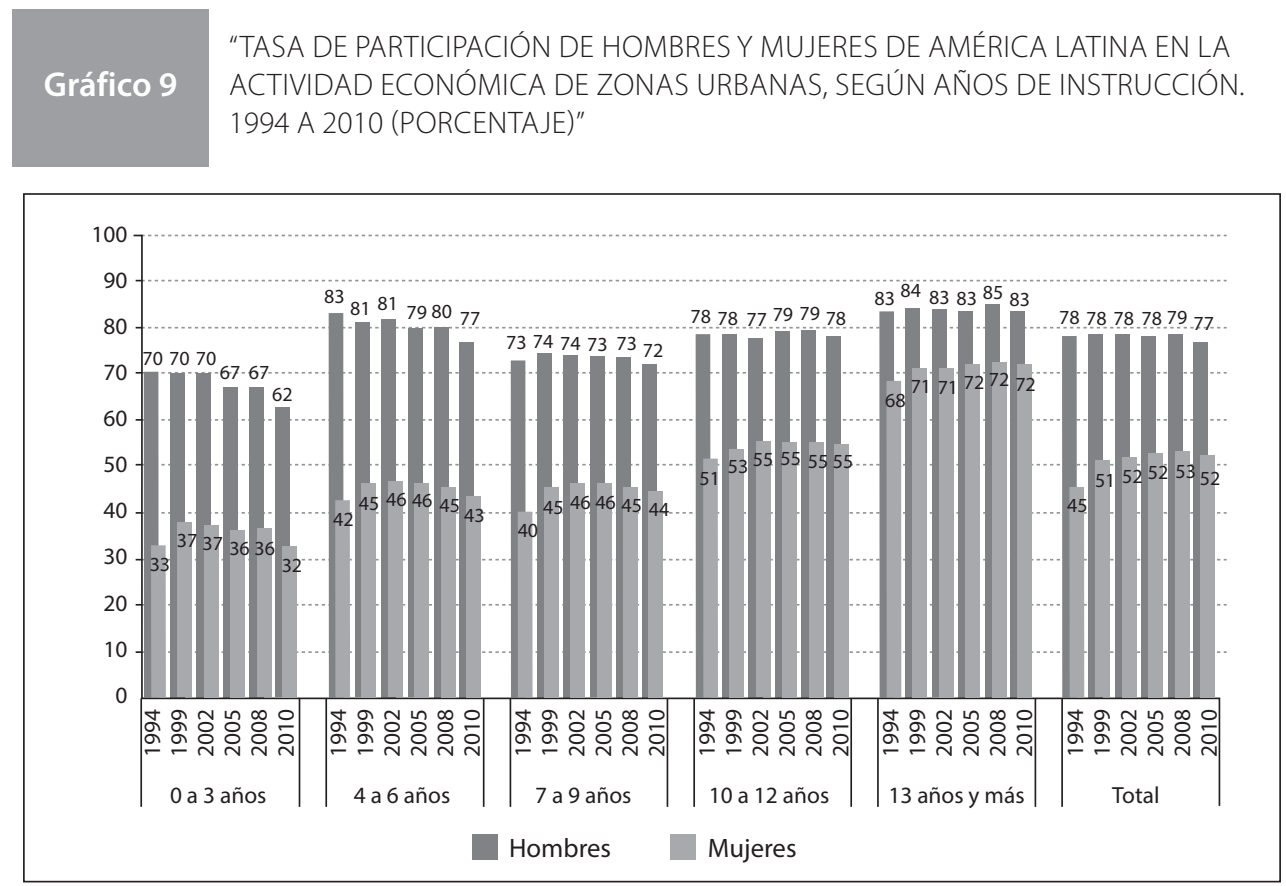

Fuente: CEPAL (2012a).

13 Este es el residual del cálculo que involucra el trabajo extradoméstico, doméstico, transporte, cuidado, arreglo personal, sueño y comidas.

14 Es posible distinguir diversas patologías en mujeres resultantes del trabajo doméstico. Algunas se expresan como daños inespecíficos como irritabilidad, dolores de cabeza, fatiga, olvidos frecuentes, angustia, temores, depresión, y otros específicos como son las enfermedades psicosomáticas, colitis, gastritis, hipertensión, migraña, neurosis, esquizofrenia y psicosis. Asimismo, pueden manifestar otros desgastes, menos objetivos que el desgaste físico. Entre ellos, la culpa frente a las demandas constantes de la familia, imposibilidad de cumplir con dos trabajos, sensación de dejar todo incompleto, sentimientos de insatisfacción frente a las pocas expectativas de triunfo y autoatribución de fracasos ajenos (Garduño, 2001). 
Destacando otros aspectos de la diferenciada participación femenina en la cúpula laboral, está la menor experiencia laboral, arraigada a la dificultosa compatibilización del embarazo y/o crianza de los hijos con el trabajo extradoméstico, y la distancia de las salas cunas para conseguirlo, principalmente, porque la "institucional laboral actual desincentiva la contratación de las mujeres y dificulta la conciliación entre la vida familiar y laboral" (Ministerio de Desarrollo Social, 2013: 106). Tales motivos oscurecen la baja tasa de participación laboral femenina en Chile, donde el año 2011 registró la más baja de América del Sur con un 52,8\% (Ministerio de Desarrollo Social, 2013).

"Las transformaciones de la vida urbana y la creciente [pero también desigual] incorporación de la mujer al mercado laboral, ha hecho más evidente que las carencias de tiempo afectan el bienestar de los hogares y, por tanto, ha crecido el interés por que esta problemática sea captada en la medición" (Damián, 2013: 144).

La autora enfatiza la importancia de incluir la dimensión temporal en los estudios de pobreza, ya que al igual que los activos y otros recursos, aparece desigualmente distribuido según género. A modo de ejemplo cuando se trabaja fuera del hogar, "después de largas jornadas de trabajo y extenuantes trayectos de transporte, difícilmente el tiempo restante (o libre) puede ser aprovechado de manera plena por los individuos para desarrollar sus capacidades y potencialidades humanas" (ibíd.,149). En nuestro país, no obstante, esta innovación no ha generado suficientes ecos para aprehender el fenómeno.

En breve, las mujeres se encuentran en una situación desventajosa en la estructura social en relación con los hombres. En ese sentido, si las mujeres, trabajen o no puertas afuera, realizan un trabajo sin horario, no remunerado, sin feriados ni vacaciones y debido a que les cuesta mucho más encontrar trabajo, tanto por la economía del cuidado como por la economía doméstica que acarrean en relación con los hombres, sin importar su nivel educativo... ¿dónde está la igualdad de la pobreza?

\section{APROXIMACIONES FINALES}

Como señala Kabeer (1980), "la pobreza como un proceso, va más allá de una fotografía instantánea" (Arriagada, 2006: 15). El monetarismo eterniza esta fotografía, al considerar solamente la privación de activos y dejando de lado una serie de aspectos íntimamente asociados a este fenómeno social. Si bien distintos enfoques han surgido para aprehender los aspectos que el monetarismo no considera, aun así, suelen ser ciegos al género.

La pobreza generalmente se equipara a reducción en los ingresos, omitiendo "la vulnerabilidad, la desigualdad, marginalidad, exclusión y discriminación" (ibíd.). Se habla de pobreza como si fuese una sola e igual para todos los individuos. No obstante para mujeres y hombres, esta es mutidimensional en sus causas y heterogénea en sus efectos. Es menester seguir ampliando la conceptualización de la pobreza, y tomar en cuenta las diferentes experiencias entre ellas y ellos, atravesadas por relaciones de poder, no solamente en el trabajo productivo remunerado, sino también en el trabajo 
reproductivo no remunerado, que sostiene y permite la circulación económica. Sin esto, es imposible aprehenderla más cabalmente.

La perspectiva de género permite comprender las desigualdades que comprometen más a las mujeres con las labores domésticas y con el cuidado de hijos y/o adultos mayores; además trae a la palestra una dimensión poco -o nada- abordada del fenómeno que es la pobreza de tiempo, la que afecta principalmente a las mujeres, tanto a las que trabajan de forma remunerada como a las que no.

"El tiempo es una categoría clave en el estudio de las relaciones de género pues proporciona evidencias empíricas sobre situaciones poco visibles relativas a la desigual distribución de trabajos y actividades de mujeres y varones" (CEPAL. 2012b: 46).

En Chile, las encuestas de uso de tiempo han tenido poco impacto y periodización, siendo la más reciente la de carácter exploratorio realizada hace cinco años por el Servicio Nacional de la Mujer (SERNAM) y el Instituto Nacional de Estadísticas (INE). Se hace urgente la necesidad de fomentar, ampliar ${ }^{15}$ e incorporar estos estudios con el propósito de complementar a los indicadores meramente monetarios utilizados, lo cual generaría una "medición" de la pobreza con una perspectiva más amplia, que implique abarcar por lo menos otras de sus dimensiones inmateriales. En efecto, permitirían seguir develando las desigualdades que se producen al interior del núcleo familiar, además permiten "seguir la evolución de la carga global de trabajo de hombres y mujeres y la situación de la división sexual del trabajo en los hogares" (ibíd.), puesto que en los desiguales usos del tiempo la pobreza también se arraiga:

El recurso temporal que "ha sido largamente reconocido", aún "no ha sido claramente reconocido como parte integral en la medición de las carencias padecidas por los hogares" (Damián, 2013: 142). Precisamente, el tiempo como fuente preponderante en su dimensión escasez/disponibilidad incide de manera importante sobre el bienestar de mujeres, hombres, pero no homogéneamente y sigue siendo eludido por las metodologías en nuestro país

Este escrito se apoya en la tesis que no existe una sola pobreza, sino varias sociales y culturales, en las que cabe mencionar la desigualdad de los quehaceres y la pobreza de tiempo. Y aunque se han desarrollado avances para contrarrestar la inequidad entre hombres y mujeres, la dominación masculina (Bourdieu, 2000) se mantiene. Los principios de visión y división siguen siendo sexuantes, tal que para muchos, mujer sigue siendo sinónimo de esposa, casa e hijos, olvidando que, en primer lugar, las mujeres son sujetos con necesidades propias, distintas a las del hogar.

Las mujeres por la atribución social de funciones reproductivas y la inserción en la estructura productiva, resultan ser más vulnerables y sobre todo, cuando son jefas de

15 Ha de suponerse, que los usos de tiempo no tendrían por qué ser los mismos entre la Región Metropolitana y el resto de las regiones del país. 
hogar (García, 2010), por las cosmovisiones esencialistas patriarcales heredadas. Pero no ha de focalizarse solamente en lo monetario para contrarrestar la carencia, sino también desventajas sociales y simbólicas, en lo que ha contribuido a perpetuar la exclusión social y la reproducción de la pobreza. Como lo indica Arriagada, se requiere de "políticas y programas que consideren el género en los procesos que originan y (que también) mantienen las situaciones de pobreza", vale decir, medidas reales que favorezcan a las mujeres "en situación más desmedrada y políticas de deconstrucción cultural que tiendan a disminuir los prejuicios valorativos" (2010: 10) sobre las mujeres.

Bajo el reconocimiento de las dispares raíces de este fenómeno social, resultado de un proceso de afectación que no es únicamente económico, sino también sociocultural, psicológico y ambiental, y que, por lo demás es dinámico y no un estado situacional, cabe esperar estrategias articuladas coherentemente para promover mayores niveles de participación, y el protagonismo de las y los actores en la solución de la precariedad. En ese sentido, y en contra de aquellas medidas de tipo universal ciegas al género que no han modificado la visión general sobre las mujeres como (potenciales) madres y al servicio de otros; abogamos a favor de medidas que se inclinen hacia la "redistribución de derechos económicos, sociales y culturales". Estas serían la clave para "brindar más autonomía y poder a las mujeres, modificando el desequilibrio de género existente y flexibilizando los roles con miras a acrecentar las opciones de hombres y mujeres" (ibíd.: 14). Esto es lo que equivaldría a pasar de un enfoque de Mujeres en Desarrollo ${ }^{16}$ al de Género y Desarrollo ${ }^{17}$ (Moser, 1995). En otras palabras, la deconstrucción de relaciones sociales que fomenten la confianza, reciprocidad y cooperación, en la sustentabilidad de iniciativas comunitarias y diversas estrategias de vida para mitigar los efectos de la pobreza. No solo en su aspecto familiar, sino también individual, por medio de las cuales las mujeres se perciban a sí mismas como sujetos libres y con poder de decisión sobre sus vidas y su entorno.

\section{BIBLIOGRAFÍA}

Arriagada, Irma (2006): "Dimensiones de la pobreza y políticas desde una perspectiva de género", en Revista de la CEPAL, № 85, [on line]. Disponible en: http: //www. eclac.org/publicaciones/xml/6/21046/lcg2266eArriagada.pdf [Recuperado el 13 de abril de 2011].

Arriagada, Irma (2010, enero 27 y 28): Capital Social, Pobreza y Género. II Seminario Internacional Género y Pobreza. CEM. México, D.F. [on line]. Disponible en: http: //cedoc.inmujeres.gob.mx/Seminarios/pobreza2010/27enero/irmaarriagada.pdf [Recuperado el 13 de abril de 2011].

Arteaga, Catalina. (2007): "Pobreza y estrategias familiares. Debates y reflexiones", en Revista Mad, No 17. p. 144-164.

16 En inglés, Women in Development (WID).

17 En inglés, Gender and Development (GAD). 
Baranda, Benito; Egenau, Paulo (2004): "Radiografía humana de la pobreza: Voces de la vulnerabilidad". Pp. 22-24, en Revista Mensaje № 374 [online] Disponible en http: // biblioteca.uahurtado.cl/ujah/msj/docs/2004/n531_22.pdf. [Recuperado el 12 de agosto 2014].

Bengoa, José (2001): "Desigualdad y seguridad ciudadana", en Revista Mensaje, Vol. 49, No 493. p. 52-55.

Bourdieu, Pierre (2000): La dominación masculina. Barcelona: Anagrama.

CEPAL (2004, enero):"Entender la pobreza desde la perspectiva de género", en Serie Mujer y Desarrollo, N052. UNIFEM. República de Italia. Santiago de Chile [on line]. Disponible en http: //www.eclac.cl/publicaciones/xml/5/14795/lcl2063e.pdf [Recuperado el 13 de abril de 2011].

(2010a, julio): ¿QuéEstado para qué igualdad? Síntesis de la Conferencia Regional sobre la Mujer de América Latina y el Caribe. Brasilia [on line]. Disponible en: http: //www.eclac.cl/publicaciones/xml/7/40117/SINTESISQue_estado_lgualdad.pdf [Recuperado el 10 de mayo de 2013].

(2010b, octubre): "Pobreza en América Latina cae en 2010 y retoma tendencia pre-crisis". [on line]. Disponible en: http://www.eclac.cl/cgi-bin/getProd.asp?xml=/ prensa/noticias/comunicados/8/41798/P41798.xml\&xsl=/prensa/tpl/p6f.xsl\&base=/ tpl/top-bottom.xslt [Recuperado el 13 de abril de 2011].

(2011): "América Latina: Evolución de la pobreza e indigencia, 1980-2011", [on line]. Disponible en: http: //www.eclac.cl/prensa/noticias/comunicados/8/45168/ grafico-evolucion-pobreza-indigencia-1980-2011-es.pdf [Recuperado el 13 de abril de 2011].

(2012a): El Estado frente a la autonomía de las mujeres. [on line]. Disponible en: http: //www.eclac.cl/publicaciones/xml/1/47381/2012-259-El_Estado_frente_autonomia_mujeres_WEB.pdf [Recuperado el 7 de mayo de 2013].

(2012b): Informe anual 2012. Los bonos en la mira, aporte y carga para las mujeres. Observatorio de Igualdad de Género de América Latina y el Caribe.[online]. Disponible en: http: //www.eclac.cl/publicaciones/xml/7/49307/2012-1042_OIGISSN_WEB.pdf [Recuperado el 7 de mayo de 2013].

(2013, diciembre) Panorama Social de América Latina. Santiago de Chile: Naciones Unidas.[on line] Disponible en: http://www.cepal.org/publicaciones/xml/9/51769/ PanoramaSocial2013.pdf[Recuperado el 30 de agosto de 2014].

Damián, Aracelly (2005): "El tiempo: la variable olvidada en los estudios de bienestar y pobreza", en Revista Sociedad y Equidad, № 5, p. 136-163. 
Feres, Juan Carlos y Mancero, Xavier (2001). "El método de las necesidades básicas insatisfechas (NBI) y sus aplicaciones en América Latina." División de Estadística y Proyecciones Económicas. Naciones Unidas, CEPAL. [on line]. Disponible en: http: //www.cepal. org/deype/mecovi/docs/taller5/8.pdf [Recuperado el 14 de agosto 2014].

García, Nancy (2010):"Reflexiones sobre la importancia de incorporar la perspectiva de género en los análisis de vulnerabilidad", en Revista La Ventana, Vol. 4. № 31. p. 7-35.

Garduño, María de los Ángeles (2001):"Para estudiar la relación entre el trabajo doméstico y la salud de las mujeres", en Revista Salud de los Trabajadores, Vol. 9. No 1. Venezuela, [on line]. Disponible en: http://www.higieneocupacional.com.br/download/trabajodomestico-salud.pdf [Recuperado el 28 de marzo de 2011].

Hartmann, Heidi (2000): "La familia como lugar de lucha política, de género y de clase: el ejemplo del trabajo doméstico", en Navarro, Marysa y Stimpson, Catharine (comp): Cambios sociales, económicos y culturales. México: FCE.

INE (2009, mayo): Encuesta Exploratoria de Uso del Tiempo en el Gran Santiago. ¿Cómo distribuyen el tiempo hombres y mujeres?, Santiago de Chile [on line]. Disponible en: http://estudios.sernam.cl/documentos/?eMTUwMTY5OA==-\%C2\%BFC\%C3\%B3mo_ Distribuyen_el_Tiempo_Hombres_y_Mujeres? [Recuperado el 26 de marzo de 2011].

Katzman, Rubén y Filgueria, Carlos (1999): Marco Conceptual sobre activos, vulnerabilidad y estructura de oportunidades. CEPAL. Uruguay. Disponible: http: //www.cepal.org/ publicaciones/xml/6/10816/LC-R176.pdf [Recuperado el 18 de agosto de 2013].

Kliksberg, Bernardo (2000): "Los Valores Culturales y su influencia en los Procesos de Desarrollo", Revista de la CEPAL. No 69. p. 85-102.

MIDEPLAN (2009, agosto): "Familias monoparentales y jefatura según sexo", en Reportes Trimestrales Situación de Género. Poblaciones Vulnerables atendidas por el Sistema de Protección Social. [on line]. Disponible en: http://siis.ministeriodesarrollosocial.gob. cl/seminario_genero2009/estad/estadistica4.html [Recuperado el 26 de marzo de 2011].

MIMDES (2005, mayo): “El Desarrollo Territorial como estrategia para la superación de la pobreza y la exclusión. Marco referencial, aspectos programáticos y experiencias en curso", Ministerio de la mujer y poblaciones vulnerables. Perú. [on line]. Disponible en: http: //www.municipioaldia.com/facipub/upload/publicaciones/1/55/modulodtrmimdes.pdf [Recuperado el 29 de abril de 2011].

Ministerio de Desarrollo Social (2012, julio 20): Resultados Mujeres, Pobreza y Trabajo. CASEN 2011 (Presentación Ministro). Santiago de Chile [on line]. Disponible en: http: // observatorio.ministeriodesarrollosocial.gob.cl/layout/doc/casen/publicaciones/2011/ CASEN_Mujeres_pobreza_y_Trabajo.pdf [Recuperado el 2 de agosto de 2012]. 
(2012b): Informe de Política Social. Pobreza y Desigualdad. Santiago de Chile. [on line] Disponible en: http: //www.ministeriodesarrollosocial.gob.cl/ipos-2012/media/ ipos_2012_pp_12-29.pdf [Recuperado el 30 de agosto de 2014].

(2013): Informe de Política Social 2013. Santiago de Chile. [on line] Disponible en: http://www.ministeriodesarrollosocial.gob.cl/ipos-2013/media/IPOS_2013.pdf [Recuperado el 16 de agosto de 2014].

(2014): Canasta Básica de Alimentos, en Observatorio Social. [on line]. Disponible en http: //observatorio.ministeriodesarrollosocial.gob.cl/ipc_pob_descripcion.php. [Recuperado el 15 de agosto 2014].

Molina, Sergio (2005): "Desigualdad en Chile: Es el tiempo de la equidad". Pp. 8-13, en Revista Mensaje No 176. Disponible en http: //biblioteca.uahurtado.cl/ujah/msj/ docs/2005/n539_08.pdf [Recuperado el 14 de agosto 2014].

Moser, Caroline (1995): Gender planning and development. Theory, practice and training. New York: Routledge.

Moser, C. (1996): Situaciones críticas. Reacción de las familias de cuatro comunidades urbanas pobres ante la vulnerabilidad y la pobreza. ESD. Serie de Estudios y monografías sobre el desarrollo ecológicamente sostenible. Estados Unidos.

Palma, Julieta y Urzúa, Raúl (2005): "Políticas contra la pobreza y ciudadanía social: el caso de Chile Solidario", en Serie de Documentos de Políticas MOST-2, Colección Políticas Sociales, No 12. UNESCO, [on line]. Disponible en: http: //unesdoc.ugnesco. org/images/0014/001402/140240s.pdf[Recuperado el 2 de mayo de 2011].

Parker, Cristián (2002): "La pobreza desde la perspectiva del desarrollo humano: desafíos para las políticas públicas en América Latina", en Cuadernos de Desarrollo Humano Sostenible, No.10. PNUD. Honduras, [on line]. Disponible en: http: //www.undp. un.hn/publicaciones/colecciones/DH_10.pdf[Recuperado el 2 de mayo de 2011].

PNUD (2010): Género: Ios desafíos para la igualdad. Programa de las Naciones Unidas para el Desarrollo Humano en Chile. Santiago de Chile: PNUD.

Rigat-Pflaum, María (2008): "Gender main streaming: un enfoque para la igualdad de género", en Revista Nueva Sociedad № 218, [on line]. Disponible en: http: //www. nuso.org/upload/articulos/3570_1.pdf [Recuperado el 28 de marzo de 2012].

Ruggeri, Caterina, Saith, Ruhi y Stewart, Frances (2003): “Does it matter that we don't agree on the definition of poverty? A comparison of four approaches", en QEH Working Paper Series, No 107, Queen Elizabeth House, University of Oxford, [on line]. Disponible en: http://www3.qeh.ox.ac.uk/pdf/qehwp/qehwps107.pdf [Recuperado el 23 de abril de 2012]. 
SERNAM (2009, febrero): "Valorización del Trabajo no remunerado (Encuesta sobre el uso del tiempo", Documento de Trabajo No 111. Departamento de Estudios y Capacitación. Santiago de Chile. Disponible en: http: //estudios.sernam.cl/ documentos/?eMTA3MDg4Mw==-Valorizaci\%C3\%B3n_del_Trabajo_Domestico_ No_Remunerado._(Encuesta_de_Uso_del_Tiempo). [Recuperado el 28 de marzo de 2012].

UNFPA (2005): Pobreza, Salud sexual y Reproductiva y Derechos Humanos. Equipo de apoyo técnico para América Latina y el Caribe. Fondo de Población de las Naciones Unidas (UNFPA) Equipo de Apoyo Técnico para América Latina y el Caribe [on line]. Disponible en: http: //venezuela.unfpa.org/doumentos/pobrezaSSRDH.pdf [Recuperado el 24 de abril de 2011]. 